\title{
Amostragem, caracterização de sintomas e escala diagramática da mancha graxa dos citros (Mycosphaerella citri) no Recôncavo Baiano
}

\author{
Sampling, characterization of citrus greasy spot (Mycosphaerella citri) symptoms in Recôncavo Baiano \\ and development of a diagrammatic scale for assessment of severity
}

\author{
Suely Xavier de Brito Silva' Francisco Ferraz LaranjeiraII Ana Cristina Fermino Soares ${ }^{\text {III }}$ \\ Sami Jorge Michereff ${ }^{\text {IV }}$
}

\section{- NOTA-}

\section{RESUMO}

O Recôncavo da Bahia é uma região de clima propício à mancha graxa dos citros (Mycosphaerella citri), doença nunca antes estudada no Brasil. Os objetivos deste trabalho foram definir o tamanho mínimo de amostra para quantificar a incidência, caracterizar os sintomas da doença e elaborar uma escala diagramática para a avaliação de sua severidade. A partir de uma amostragem piloto realizada por avaliação de cinco folhas por quadrante, quatro quadrantes por planta e 30 plantas em cada um dos 10 pomares visitados no município de Cruz das Almas, foi determinado que o tamanho mínimo da amostra em quadrantes e folhas seria de quatro e 16 plantas, respectivamente. De 320 folhas coletadas de diferentes plantas em 11 pomares, foi constatada uma grande quantidade de lesões (média de 131) por folha sintomática, em sua maioria diminutas (média de $0,014 \mathrm{~cm}^{2}$ ). A severidade variou de 0,15\% a 35,85\%, com média de 7,3\%. Com base na severidade real em campo, foi elaborada uma escala diagramática com seis níveis: $1 \%, 2 \%, 5 \%, 9 \%, 18 \%$ e $36 \%$ de área foliar lesionada. Cinqüenta imagens de folhas foram submetidas a avaliadores por duas vezes, os quais estimaram a severidade com e sem a utilização da escala, constatando-se um discreto benefício no grau de precisão e de acurácia das estimativas com o uso da escala.

Palavras-chave: Mycosphaerella citri epidemiologia, severidade, Bahia.

\section{ABSTRACT}

Weather conditions make the Recôncavo Region in the State of Bahia, a favorable region to the occurrence of citrus greasy spot (Mycosphaerella citri), a disease that hadn't been studied before in Brazil. Thus, this research aimed to develop a sampling method to quantify its incidence, to characterize its symptoms, and to develop a diagrammatic scale for assessing disease severity. The minimum sample size to estimate greasy spot incidence in leaves (16 plants) was calculated based on a prospective sampling of five leaves per quadrant, four quadrants per tree, and 30 trees per grove, in ten groves. From 320 mature leaves collected in different plants of 11 groves, it was observed a high number of lesions per symptomatic leaf (mean of 131 102 ), most of them small (mean of $0.014 \pm ., 011 \mathrm{~cm}^{2}$ ). Greasy spot severity varied from $0,15 \%$ to $35,85 \%$, with a mean of $7,3 \%$. Based on those severity values, a six level (1\%, 2\%, 5\%, 9\%, 18\% e 36\% of lesioned leaf area) diagrammatic scale was developed. 50 images of symptomatic leaves were showed to evaluators, which estimated the severity with and without the use of the scale. The diagrammatic scale use, slightly increased the estimates accuracy and precision.

Key words: Mycosphaerella citri, epidemiology, severity, Bahia.

A mancha graxa dos citros (Mycosphaerella citri) afeta frutos e folhas, podendo depreciar o produto final e promover severa desfolha, levando a danos de até 45\% na produção (TIMMER \& GOTTWALD, 2000). A doença está relacionada à umidade relativa do ar próxima de $100 \%$, associada a períodos de altas temperaturas (TIMMER \& GOTTWALD, 2000). Na Bahia, a região do Recôncavo possui clima propício à enfermidade, resultando em sintomas foliares típicos:

'Agência Estadual de Defesa Agropecuária da Bahia (ADAB), Universidade Federal do Recôncavo da Bahia (UFRB), Cruz das Almas, BA, Brasil.

IIEmbrapa Mandioca e Fruticultura Tropical, CP 007, 44380-000, Cruz das Almas, BA, Brasil. E-mail: chico@cnpmf.embrapa.br.

*Autor para correspondência.

IILLaboratório de Fitopatologia, UFRB, Cruz das Almas, BA, Brasil.

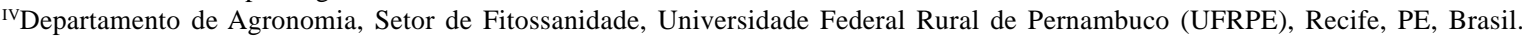


lesões escuras de aspecto oleoso, com margens difusas e halo amarelo-esverdeado (TIMMER \& GOTTWALD, 2000). A doença ocorre no Brasil, mas sempre foi considerada de importância menor (LARANJEIRA et al., 2005). Talvez por isso não existem estudos a seu respeito no país.

Para quantificação de doenças, as plantas devem ser amostradas criteriosamente a fim de que a amostra seja representativa da população original (AMORIM, 1995). Em qualquer sistema amostral, a amostragem piloto é fundamental, pois é nela que dados são coletados para obter-se a variação da característica estudada, possibilitando o cálculo da suficiência amostral (tamanho da amostra). O objetivo do levantamento, o modelo de dispersão da doença, a disponibilidade de tempo, os recursos e os níveis de precisão e acurácia são variáveis que influenciam o tamanho da amostra (AMORIM, 1995). Mesmo no exterior não são encontrados trabalhos de amostragem para estimativa de incidência ou severidade da MGC. Na Costa Rica, foram quantificadas incidência e a severidade por meio da adaptação de uma escala diagramática. Obteve-se a estimativa da área com sintomas de MGC (HIDALGO et al., 1997), mas os autores não relataram estudos prévios de amostragem para definição do tamanho de amostras.

Apenas quando uma doença é mensurada corretamente é que se pode demonstrar o quanto de dano ela ocasiona. No caso de sintomas como manchas, a percentagem de área de tecido afetado (severidade) geralmente retrata melhor a quantidade de doença que a incidência (AMORIM, 1995). Entre as estratégias para quantificação da severidade, destacam-se as escalas diagramáticas, que são representações de plantas ou suas partes com sintomas em diferentes níveis de severidade (AMORIM, 1995). Na sua elaboração devem ser considerados os limites inferiores e superiores que devem corresponder, respectivamente, à mínima e à máxima quantidade de doença encontrada no campo; a plotagem dos sintomas, devendo estar o mais próximo possível daqueles observados na planta; e os níveis intermediários da severidade da doença, atentando para as limitações de acuidade da visão humana (AMORIM, 1995).

Este trabalho teve como objetivos: determinar o tamanho mínimo de amostras para quantificar a incidência da doença, caracterizar a sintomatologia da MGC no Recôncavo Baiano e elaborar uma escala diagramática para a avaliação da severidade da doença.

A amostragem piloto foi realizada em maio de 2006, quando 10 pomares no município de Cruz das Almas, Bahia, foram avaliados por duas pessoas previamente treinadas no reconhecimento de sintomas da MGC. As avaliações foram estratificadas em plantas, quadrantes (quatro por planta) e folhas maduras (cinco por quadrante); o caminhamento seguiu padrão em "W" avaliando-se 30 plantas dentro de cada pomar. Determinou-se o número mínimo de plantas por pomar para estimar a incidência, usando-se a equação:

$e=t_{(\alpha / 2 ; n-1)} \frac{S}{\sqrt{n}}$, em que: e = erro admitido para a amostragem (5\%); $\mathrm{t}$ = valor tabelado da distribuição de t com nível de significância $\alpha$ de $1 \%$ e $n-1$ graus de liberdade; s = desvio padrão amostral; $\mathrm{n}$ = tamanho da amostra (COCHRAN, 1965).

Nos mesmos pomares foram coletadas 320 folhas de laranja-doce 'Pêra' (Citrus sinensis Osb.), uma folha por árvore, com sintomas típicos. As folhas foram digitalizadas e, com auxílio do programa Assess ${ }^{\circledR}$, foram determinadas a área foliar total e a área lesionada, o número de lesões e a severidade da doença em cada folha. Nesse processo, foi usada a face inferior das folhas, desconsiderando-se o halo amarelado das lesões, comum na face superior, e que em testes preliminares interferiu de maneira aleatória na determinação da severidade.

Para a elaboração da escala diagramática com níveis de severidade (1\%, 2\%, 5\%, 9\%, 18\% e 36\%) (Figura 1A), foram utilizados os dados e as imagens obtidos na caracterização dos sintomas. Para a validação da escala, 50 imagens contendo os sintomas da MGC foram selecionadas e inseridas em slides individuais para visualização em Power Point. Na primeira etapa, 32 avaliadores com experiência variada na quantificação de doenças estimaram a severidade das 50 imagens e, um mês após, repetiram a avaliação com o auxílio da escala diagramática. Pela regressão linear da severidade real $\mathrm{x}$ a severidade estimada pelos avaliadores, foram determinadas a acurácia e a precisão de cada um deles. A acurácia foi determinada pelo teste t, a 5\% de probabilidade de erro, aplicado ao intercepto da regressão linear (a), verificando-se se $a \neq 0$, e ao coeficiente angular da reta (b), testando-se se $b \neq 1$. Valores de $a \neq 0$ indicam a ocorrência de constantes desvios, enquanto valores de $b \neq 1$ indicam a presença de desvios sistemáticos (NUTTER et al., 1993). A precisão das estimativas foi obtida pelos coeficientes $\left(r^{2}\right)$ e pelo erro padrão da regressão.

A amostragem piloto detectou $100 \%$ dos pomares e plantas com sintomas da doença. Assim, para estudos de incidência de pomares ou de plantas afetadas, o número de amostras pode ser o mínimo possível, ou seja, um pomar e uma planta. Por outro lado, uma proporção de $0,973 \pm 0,04$ dos quadrantes e $0,442 \pm 0,11$ das folhas apresentaram sintomas. Assim, calculou-se em quatro e 16 plantas o número mínimo para estimativa da incidência da MGC em quadrantes e em folhas, respectivamente. O baixo número de amostras requeridas é implicação direta da alta incidência, mas também da baixa variação entre 

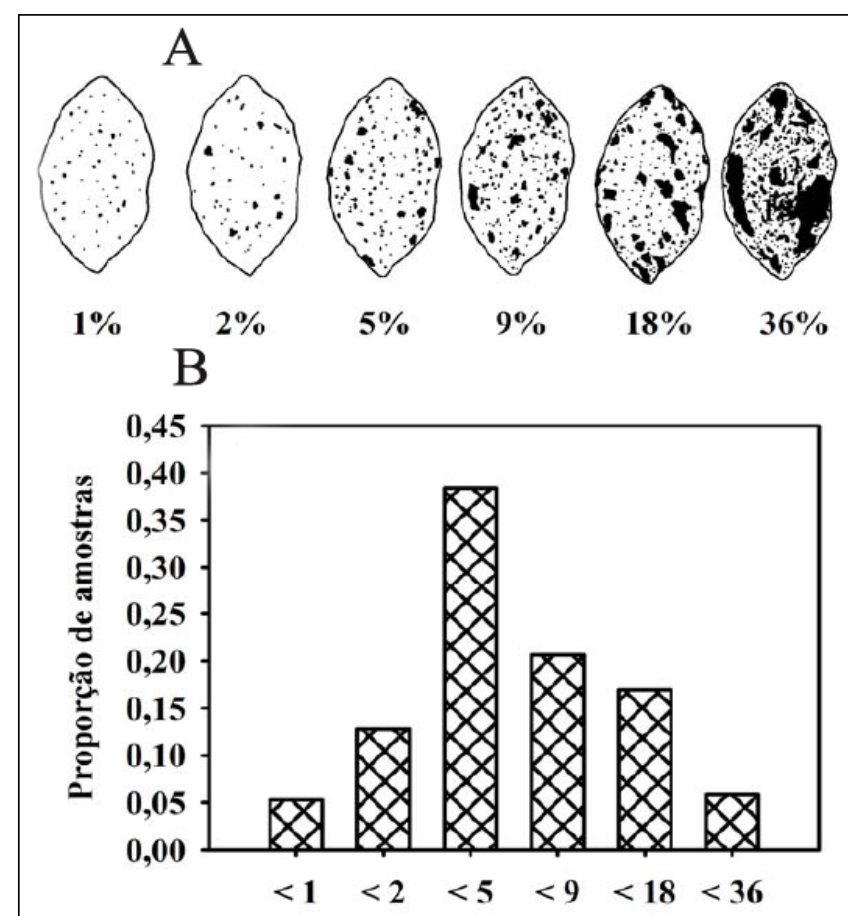

Severidade (\% de área foliar lesionada)

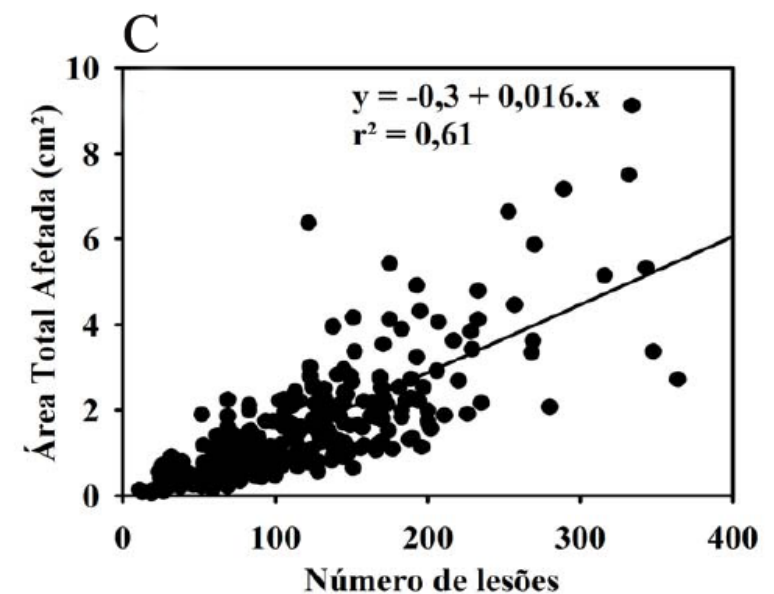

Figura 1 - (A) Escala diagramática preliminar da mancha graxa dos citros, com seis níveis de severidade; (B) Distribuição, em proporção de amostras, da severidade real de mancha graxa dos citros; (C) Relação do número de lesões por área foliar total afetada $\left(\mathrm{cm}^{2}\right)$ em folhas de laranja 'Pêra', Cruz das Almas, BA.

unidades amostrais. Essa constatação sustenta a hipótese de que a MGC esteja amplamente disseminada, contrastando com o fato de ser considerada uma doença secundária na citricultura.

Os sintomas observados foram idênticos aos descritos na literatura (HIDALGO et al., 1997), ou seja, lesões foliares de aspecto oleoso, variando do marrom brilhante ao preto, com margens difusas, envoltas em halo amarelo-esverdeado. Notouse que nem sempre o halo podia ser identificado e que na face inferior das folhas ele não ocorria. As lesões foram observadas em ambas as faces, mas na face superior apenas as maiores lesões eram percebidas. Na inferior, ao contrário, lesões de distintos tamanhos eram notadas. Mais de $50 \%$ das amostras apresentaram menos de 5\% de área foliar lesionada (Figura 1B). A severidade máxima observada foi de $35,85 \%$, pois é comum a ocorrência de desfolha. A severidade mínima foi de $0,15 \%$, com média de $7,3 \%$ e mediana de 10\% (Figura 1B). Situação similar foi encontrada por HIDALGO et al. (1997) na Costa Rica, que relataram severidade média de até $5 \%$.

O número de lesões por folha teve como extremos os valores de 1.078 e 11 , com média de 131 e mediana de 114 , sendo que $78,7 \%$ das folhas apresentaram até 200 lesões. Constatou-se uma variação entre 0,0025 e $0,084 \mathrm{~cm}^{2}$ para tamanho médio das lesões, com média de $0,014 \mathrm{~cm}^{2}$ e mediana de $0,012 \mathrm{~cm}^{2}$. Das folhas amostradas, a área média de lesão foi inferior a $0,02 \mathrm{~cm}^{2}$ para $81 \%$ das folhas. Podese considerar como alto o número de lesões por folha, mas baixa a área ocupada por cada lesão. Isso explica a boa relação entre o número de lesões e a área foliar total afetada (Figura 1C). De acordo com TIMMER \& GOTTWALD (2000), a área lesionada é função do crescimento epifítico do fungo. Assim sendo, infere-se que o padrão observado denota uma grande densidade de inóculo depositada nas folhas, mas de crescimento epifítico limitado ou ocorrência de competição por sítios de infecção. Essa hipótese é corroborada pelos resultados da amostragem piloto.

Menos da metade dos avaliadores apresentou desempenho superior ao usarem a escala (Tabela 1). Isso indica que é necessária uma seleção e um treinamento prévio dos avaliadores quanto à quantificação de sintomas e ao uso específico da escala para MGC. Dos avaliadores com desempenho melhorado (Tabela 1), a maioria não tinha experiência em quantificação de sintomas, resultando em regressões não significativas e coeficientes de regressão $\left(\mathrm{r}^{2}\right)$ muito baixos na primeira avaliação. Entre as duas etapas observou-se um incremento na precisão das estimativas e uma diminuição no erro padrão das regressões. A maioria dos avaliadores tendeu a subestimar a doença, fato comprovado pelos valores de (b), significativamente menores que um para 10 avaliadores na primeira avaliação e para oito, na segunda (Tabela 
Tabela 1 - Análise de regressão linear simples para estimativas da severidade da mancha graxa em duas avaliações, a primeira sem o uso da escala diagramática e a segunda com a escala diagramática. Intercepto (a), coeficiente angular da reta (b), coeficiente de determinação $\left(r^{2}\right)$ e erro padrão obtidos pela regressão linear entre a severidade real (variável independente) e a severidade estimada (variável dependente).

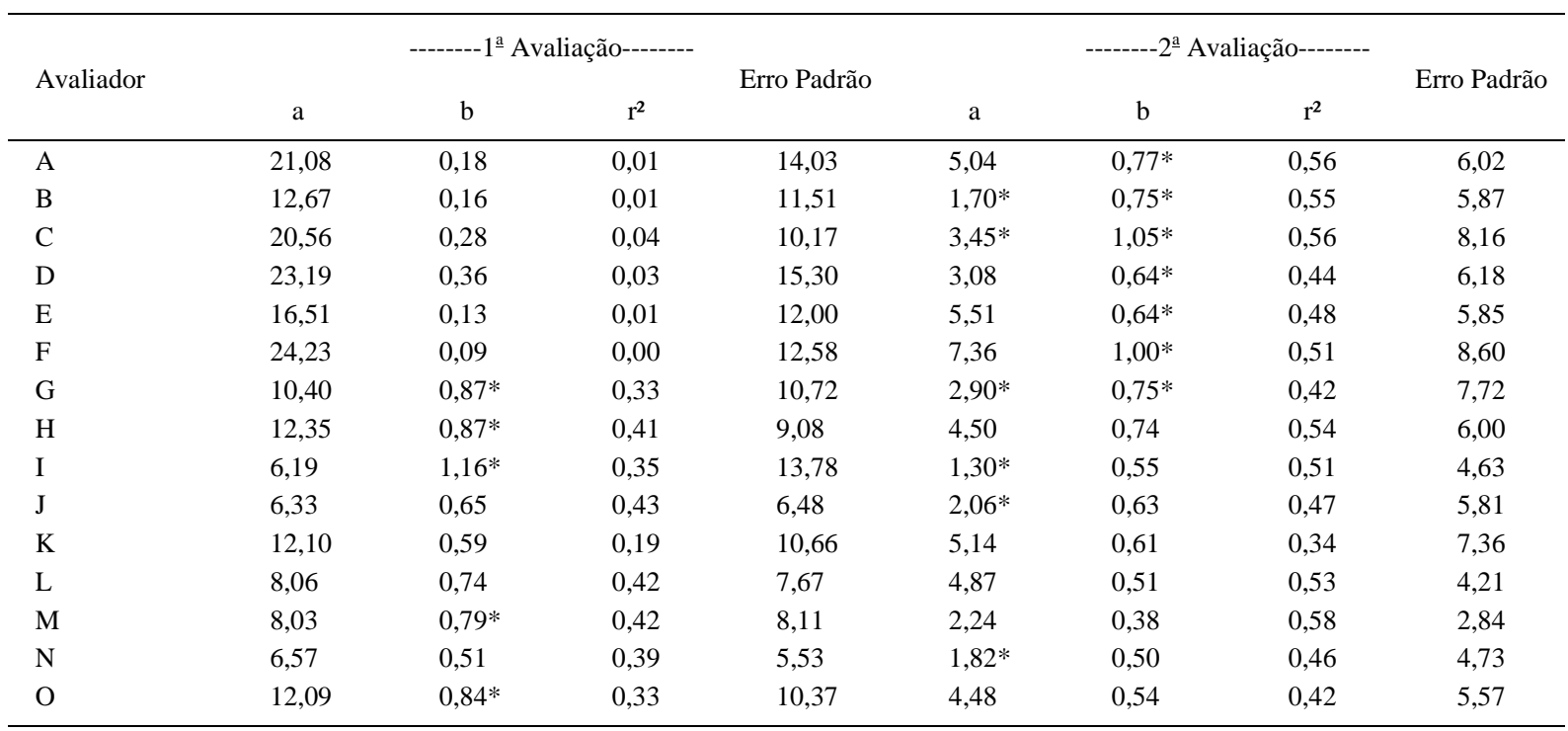

* Valores de a e b marcados com asterisco não diferem de 0 e 1, respectivamente, pelo teste t a $5 \%$ de probabilidade de erro.

1). O uso da escala levou a menores valores médios da interseção (a), com 40\% dos avaliadores apresentando valores de (a) indistintos de zero na segunda avaliação (Tabela 1). Isso mostra que o uso da escala proporcionou menos desvios constantes, além de incremento na acurácia dos avaliadores. Neste trabalho não foi possível identificar causas únicas para o sucesso apenas modesto da escala. Uma possibilidade seria a falta de treinamento dos avaliadores. No entanto, avaliadores experientes não estimaram bem a severidade, com ou sem o auxílio da escala. Esse fato põe em dúvida a capacidade humana em distinguir algumas lesões causadas pela MGC. Como além de muito pequenas elas são numerosas, ficaria difícil para o avaliador, mesmo com o uso da escala, condensar todo estímulo visual numa estimativa numérica. Isso contrasta com a precisão do programa utilizado para quantificação da severidade real que, possivelmente, identifica tecido lesionado que seja ilegível ao alcance da visão humana. Para que uma escala diagramática possa ser usada na estimativa da severidade da MGC, serão necessários ajustes na escala e, portanto, a escala apresentada neste trabalho deve ser considerada preliminar. Além disso, para elevar o grau de confiabilidade e de repetibilidade dos resultados, será necessária a seleção prévia de avaliadores.

Até onde se pôde levantar, este é o primeiro trabalho com mancha graxa dos citros no Brasil. Por seu caráter pioneiro, os resultados obtidos são referentes a aspectos básicos, que servirão como fundamento para trabalhos mais específicos.

\section{AGRADECIMENTOS}

À Agência Estadual de Defesa Agropecuária da Bahia (ADAB), pelo apoio financeiro e ao Sr. Décio de Oliveira Almeida, pela assistência na coleta e na análise dos dados. Os autores FFL, ACFS e SJM são bolsistas em produtividade em pesquisa do Conselho Nacional de Desenvolvimento Científico e Tecnológico (CNPq).

\section{REFERÊNCIAS}

AMORIM, L. Avaliação de doenças. In: BERGAMIN FILHO, A. et al. (Ed.). Manual de fitopatologia. 3.ed. São Paulo: Agronômica Ceres, 1995. Cap.32, p.647-671.

COCHRAN, W.G. Técnicas de amostragem. Rio de Janeiro: Fundo de Cultura, 1965. 555p.

HIDALGO, H. et al. Epidemiology and control of citrus greasy spot on Valencia orange in the humid tropics of Costa Rica. Plant Disease, St. Paul, v.81, n.9, p.1015-1022, 1997.

LARANJEIRA, F.F. et al. Fungos, procariotos e doenças abióticas. In: MATTOS JÚNIOR, D. et al. (Ed.). Citros. Campinas: Instituto Agronômico / Fundag, 2005. Cap 18, p.511558.

NUTTER JR., F.W. et al. Assessing the accuracy, intra-rater repeatability, and inter-rater reliability of disease assessment systems. Phytopathology, St. Paul, v.83, n.8. p.806-812, 1993.

TIMMER, L.W.; GOTTWALD, T.R. Greasy spot and similar diseases. In: TIMMER, L.W. et al. (Ed.). Compendium of citrus diseases. 2.ed. St. Paul: APS s, 2000. p.25-29. 